\title{
The Finite Volume Method an Alternative Method for LF Electromagnetic Problems
}

\author{
Riad Benghalia*, Ahmed Cheriet, Ishaq Amrani
}

LGEB Laboratory, Electrical Engineering Department, Biskra University, Biskra 07000, Algeria

Corresponding Author Email: a.cheriet@univ-biskra.dz

https://doi.org/10.18280/ejee.224-507

Received: 25 May 2020

Accepted: 16 September 2020

\section{Keywords:}

$3 D$ triangular mesh, FVM, force, torque, dynamics

\begin{abstract}
Currently, many simulation tools based on numerical methods are available for modelling of low frequency electromagnetic problems such as eddy current related problems, electrical machines and electromagnetic actuators analysis. Commonly, it's the finite element method (FEM) which is used; nevertheless, the exploit of other numerical approaches, such as the finite volume method (FVM) can be strongly promising. Accordingly, the main purpose of this paper is to present the FVM method as an alternative method for low frequency electromagnetic problems. Thus, 2D and 3D FVM computer codes are developed and examined through the analysis of two TEAM workshop problems and an experimental electromagnetic micro-actuator. These types of problems are habitually analyzed by the FEM method. By using the FVM method, the solution of the above listed problems includes eddy current, torque and magnetic force computation.
\end{abstract}

\section{INTRODUCTION}

Computational electromagnetic tools are impressively indispensable for analysing and optimization of electromagnetic devices such as electrical machines. These numerical tools help powerfully designers in order to minimize both time and cost. At the present time, many simulation tools based on numerous numerical methods are commercially available even for low (LF) or high (HF) frequency electromagnetic problems. For LF electromagnetic problems such as related to eddy current analysis or magnetic force computation, it is the finite element method (FEM) which is commonly used according to its efficiency. What's more, because the method is enough developed and famously known throughout the scientific community in electromagnetic. However, there exist other numerical methods which can be as well extremely promising for LF electromagnetic problems, such as the finite volume method (FVM). Several previous works have been interested in the comparison between the FVM and the FEM regarding various physical problems [1-4]. Compared to the FEM, the FVM method is especially promising in terms of computational time (CPU) and required storage memory [5-7]. Originally, the FVM method is applied for the solution of problems related to fluid flows $[8,9]$. Then, the FVM method is applied to solve Maxwell's equations in the HF domain such as in wave propagation problems $[10,11]$. The method is applied moreover to solve some electrostatic problems such as in the study [12]. In the last few years, the FVM is presented as a new method to solve LF electromagnetic problems $[13,14]$ where quasi-static magnetic field is analysed. As well, other researchers are interesting in developing FVM in time domain to analyze nonlinear electromagnetic field [15].

For 3D LF electromagnetic problems, note that the FVM method has been already developed where a simple hexahedral volume element is used [16, 17] (Figure 1.a). Thus, in order to handle more complex geometries, this paper proposes a $3 \mathrm{D}$ unstructured FVM by making use of prism element (Figure 1.b).

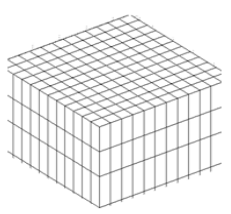

(a)

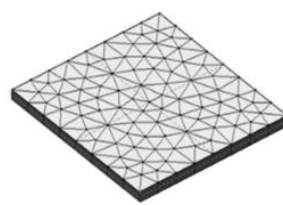

(b)
Figure 1. Control volume shape for 3D FVM method (a) Hexahedral mesh, (b) Prism mesh

As well, for 2D LF electromagnetic problems, a simple rectangular control volume is usually associated to the FVM [18]. Furthermore, to give more flexibility to the FVM to analyze more complex geometries in the 2D case, this paper proposes in addition a 2D unstructured FVM by making use of triangular mesh.

To check the developed computing codes, two TEAM workshop problems and an experimental electromagnetic micro-actuator are analyzed. The solution of $1^{\text {st }}$ TEAM includes the determination of levitation height of an aluminium plate and the applied magnetic force, while the $2^{\text {nd }}$ one requires torque computation. The two TEAM problems require eddy current computation for either force or torque computation. The third problem is related to magnetic force computation and dynamic analyses.

\section{FVM FORMULATION}

To give an idea as well as more details on the FVM method, in this section we will study the FVM in both cases 2D with triangular element and 3D with prism volume. For both cases, starting from Maxwell's equations [19], in terms of the 
magnetic vector potential $\mathbf{A}$, the equation to solve is:

$$
\begin{gathered}
\nabla \times \frac{1}{\mu} \nabla \times \mathbf{A}=\mathbf{J}_{\mathrm{s}}+\mathbf{J}_{\text {ind }} \\
\mathbf{A}=0 \text { at infinity }
\end{gathered}
$$

$\mu$ is the magnetic permeability, $\mathbf{J}_{s}$ is the source current density and $\mathbf{J}_{\text {ind }}$ is the eddy current density. Eq. (2) describes the impressed Dirichlet condition at infinity.

\subsection{D case}

In the 2D case the magnetic flux density is defined only in the Oxy plan and consequently the magnetic vector potential and the source current density have only one component as:

$$
\begin{aligned}
\mathbf{A} & =A_{z} \mathbf{k} \\
\mathbf{J}_{s} & =J_{s z} \mathbf{k}
\end{aligned}
$$

where, $\mathbf{k}$ is the unit vector in the $z$ direction. Thus, in this case Eq. (1) can be rewritten as:

$$
-\nabla \cdot\left(\frac{1}{\mu} \nabla A_{z}\right)=J_{s z} \mathbf{k}+\mathbf{J}_{\text {ind }}
$$

The eddy current density, which is induced either by movement or temporal variation of the current source, is given by:

$$
\mathbf{J}_{\text {ind }}=\sigma\left(-\frac{\partial A_{z}}{\partial t} \mathbf{k}+\mathbf{v} \times \mathbf{B}\right)
$$

In Eq. (6) $\mathbf{v}$ is the velocity, B is the magnetic flux density and $\sigma$ is the electrical conductivity. In the 2D case; the velocity term can be rewritten as follows:

$$
\mathbf{v} \times \mathbf{B}=-\left(v_{x} \cdot \frac{\partial A_{z}}{\partial x}+v_{y} \cdot \frac{\partial A_{z}}{\partial y}\right) \mathbf{k}
$$

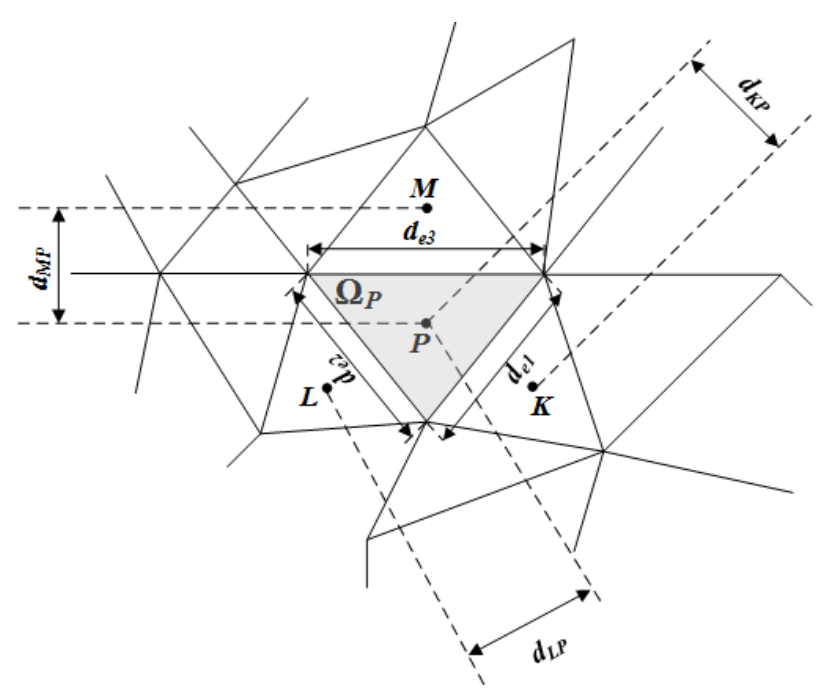

Figure 2. Triangular mesh scheme for 2D FVM method $v_{x}$ and $v_{y}$ are the velocity components in the Oxy plan. By substituting Eq. (7) in Eq. (6), and by using the harmonic representation, Eq. (5) becomes:

$$
-\nabla \cdot\left(\frac{1}{\mu} \nabla A_{z}\right)+\sigma\left(j \omega A_{z}+v_{x} \cdot \frac{\partial A_{z}}{\partial x}+v_{y} \cdot \frac{\partial A_{z}}{\partial y}\right) \mathbf{k}=J_{s z} \mathbf{k}
$$

At this stage, Eq. (8) has to be solved by the 2D FVM method with triangular mesh. As described in Figure 2, in the case of triangular mesh scheme, each principal control volume $\Omega_{p}$ is represented by its principal node $P$ and surrounded by three neighbourhood control volumes represented by their nodes $K, L$ and $M$.

Subsequently, the FVM method involves a surface integration of Eq. (8) over the principal control volume $\Omega_{p}$ of node $P$ as:

$$
\begin{aligned}
& \iint_{\Omega_{p}}-\nabla \cdot\left(\frac{1}{\mu} \nabla A_{z}\right) \mathrm{d} \Omega+\iint_{\Omega_{p}} \sigma \omega A_{z} \mathrm{j} \mathrm{d} \Omega \\
& +\iint_{\Omega_{p}} \sigma\left(v_{x} \cdot \frac{\partial A_{z}}{\partial x}+v_{y} \cdot \frac{\partial A_{z}}{\partial y}\right) \mathrm{d} \Omega=\iint_{\Omega_{p}} J_{s z} \mathrm{~d} \Omega
\end{aligned}
$$

The first term in Eq. (9) can be rewritten as follows:

$$
\begin{aligned}
\iint_{\Omega_{p}}-\nabla \cdot\left(\frac{1}{\mu} \nabla A_{z}\right) \mathrm{d} \Omega & =-\frac{1}{\mu} \underset{\partial \Omega_{p}}{\oint} \nabla A_{z} \cdot \mathbf{n} \cdot \mathbf{d l} \\
& =-\frac{1}{\mu} \sum_{i=1}^{3} \nabla A_{z} \cdot \mathbf{n}_{i} \cdot d_{e i}
\end{aligned}
$$

$\mathrm{n}$ is the normal vector and $d_{e i}$ is the $\mathrm{i}^{\text {th }}$ edge length with respect to $i=1,2,3$. The term $\nabla A_{z} \cdot \boldsymbol{n}_{i}$ can be approximated for each edge by a finite difference, for example for the edge $d_{e l}$ :

$$
\left(\nabla A_{z} \cdot \mathbf{n}_{1}\right)_{d e 1}=\frac{A_{z}^{K}-A_{z}^{P}}{d_{K P}}
$$

By the way, Eq. (10) leads to an algebraic expression as:

$$
\begin{array}{r}
-\frac{1}{\mu} \sum_{i=1}^{3} \nabla A_{z} \cdot \mathbf{n}_{i} \cdot d_{e i}=-\frac{1}{\mu}\left[\frac{A_{z}^{K}-A_{z}^{P}}{d_{K P}} \cdot d_{e 1}\right. \\
\left.+\frac{A_{z}^{L}-A_{z}^{P}}{d_{L P}} \cdot d_{e 2}+\frac{A_{z}^{M}-A_{z}^{P}}{d_{M P}} \cdot d_{e 3}\right]
\end{array}
$$

Eq. (12) can be rewritten as follows:

$$
\begin{array}{r}
-\frac{1}{\mu} \sum_{i=1}^{3} \nabla A_{z} \cdot \mathbf{n}_{i} \cdot d_{e i}=\frac{1}{\mu}\left(\frac{d_{e 1}}{d_{K P}}+\frac{d_{e 2}}{d_{L P}}+\frac{d_{e 3}}{d_{M P}}\right) \cdot A_{z}^{P} \\
-\frac{1}{\mu}\left(\frac{d_{e 1}}{d_{K P}} \cdot A_{z}^{K}+\frac{d_{e 2}}{d_{L P}} \cdot A_{z}^{L}+\frac{d_{e 3}}{d_{M P}} \cdot A_{z}^{M}\right)
\end{array}
$$

Let's assume a uniform distribution of the current density in the principal control volume $\Omega_{p}$. Therefore, the source term is integrated as follows: 


$$
\iint_{\Omega p} J_{s z} d \Omega=J_{s z P} \cdot S_{\Omega p}
$$

The integration of all terms given by Eq. (9) leads in an algebraic equation that express the potential in the principal control volume in terms of potentials of its three neighbourhoods as:

$$
A_{z}^{P}=\frac{1}{C_{p}}\left(C_{\mathrm{k}} A_{z}^{K}+C_{1} A_{z}^{L}+C_{\mathrm{m}} A_{z}^{M}+J_{\mathrm{szP}} \cdot S_{\Omega p}\right)
$$

The coefficients $C_{p}, C_{k}, C_{l}$ and $C_{m}$ describe geometrical and physical properties associated with the principal control volume and its neighbourhoods. $\mathrm{J}_{s z P}$ is the current density in the principal control volume. Rewriting Eq. (15) for all control volumes, thus leads to the following matrix equation:

$$
\left[\mathbf{M}_{2}\right]\left[A_{z}\right]=\left[s_{s z}\right]
$$

Compared to the FEM method, we point out that the FVM method leads to a sparser matrix $\mathbf{M}_{2}$; this particular property are very significant regards to computational time and required storage memory.

\subsection{D case}

The 3D standard FVM method featured by hexahedral control volume shape has been already demonstrated in previous works showing its effectiveness for electromagnetic devices. Unfortunately, the hexahedral element appears too cost in the case of complex geometry, such as for example for circular coils. To overcome the limitation of the standard FVM method and enable it to handle more complex geometry, we propose a $3 \mathrm{D}$ unstructured FVM method. In such alternative the simple hexahedral control volume is replaced by a prism control volume (Figure 3).

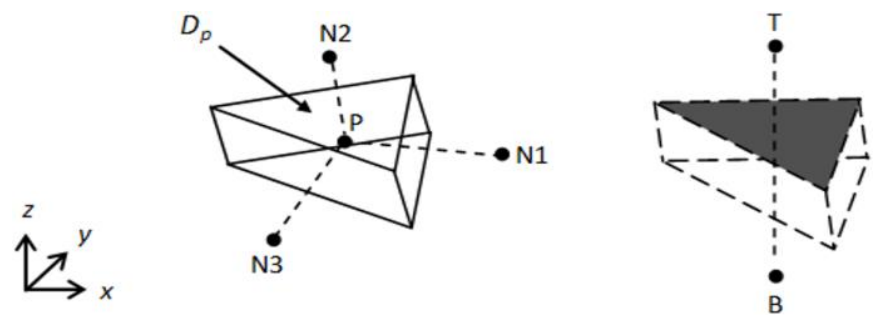

Figure 3. Prism control volume for 3D FVM method

Here, each control volume element $D_{p}$ consists of a central node $P$ and delimited by five faces. The neighbourhood control volumes of $D_{p}$ are represented by their central nodes $K$, $L$ and $M$ in the Oxy plan, $B$ and $T$ according to the $z$ direction. As in the 2D case, Eq. (1) must be integrated over the prism control volume:

$$
\int\left[\iint_{\Omega_{p}}\left(\nabla \times \frac{1}{\mu} \nabla \times \mathbf{A}\right) d \Omega_{p}\right] d z=\int\left[\iint_{\Omega_{p}}\left(\mathbf{J}_{\mathrm{s}}+\mathbf{J}_{\text {ind }}\right) d \Omega_{p}\right] d z
$$

with:

$$
d \Omega_{p} d z=d D_{p}
$$

According to the Green-Ostrogradsky theorem, Eq. (17) becomes:

$$
-\iiint\left(\nabla \cdot\left(\frac{1}{\mu} \nabla \mathbf{A}\right)\right) \mathrm{d} D_{p}=\iiint_{D p}\left(\mathbf{J}_{\mathrm{s}}+\mathbf{J}_{\text {ind }}\right) \mathrm{d} D_{p}
$$

For the first term we have:

$$
-\iiint_{D p} \nabla \cdot\left(\frac{1}{\mu} \nabla A\right) d D_{p}=-\oiint_{\mathrm{S}} \frac{1}{\mu} \nabla A \cdot d \mathbf{s}
$$

Let consider for example the integration of the $x$ component:

$$
\begin{gathered}
-\oiint \frac{1}{\mu} \nabla A_{x} \cdot d s \cdot \mathbf{n}=-\sum_{\mathrm{i}=1}^{5} \frac{1}{\mu_{i}} \nabla A_{x} \cdot d \mathbf{s}_{i} \\
=-\left[\frac{S_{1}}{\mu_{1}} \frac{A_{x 1}-A_{x p}}{d l_{1} \sin \left(d e_{1}, d l_{1}\right)}+\frac{S_{2}}{\mu_{2}} \frac{A_{x 2}-A_{x p}}{d l_{2} \sin \left(d e_{2}, d l_{2}\right)}\right. \\
+\frac{S_{3}}{\mu_{3}} \frac{A_{x 3}-A_{x p}}{d l_{3} \sin \left(d e_{3}, d l_{3}\right)}+\frac{S_{4}}{\mu_{4}} \frac{A_{x 4}-A_{x p}}{d l_{4} \sin \left(d e_{4}, d l_{4}\right)} \\
\left.+\frac{S_{5}}{\mu_{5}} \frac{A_{x 5}-A_{x p}}{d l_{5} \sin \left(d e_{5}, d l_{5}\right)}\right]
\end{gathered}
$$

Let us define the first coefficient as:

$$
C_{1}=\frac{S_{1}}{\mu_{1}} \frac{1}{d l_{1} \sin \left(d e_{1}, d l_{1}\right)}
$$

Subsequently, all the coefficients in the previous algebraic equation can be rewritten as the the following general form:

$$
C_{i}=\frac{S_{i}}{\mu_{i}} \frac{1}{d l_{i} \sin \left(d e_{i}, d l_{i}\right)}
$$

The developpement of the $x$ component of the first term leads to the following algebraic equation:

$$
\begin{aligned}
& -\left(C_{1}\left(A_{x 1}-A_{x p}\right)+C_{2}\left(A_{x 2}-A_{x p}\right)+C_{3}\left(A_{x 3}-A_{x p}\right)\right. \\
& \left.+C_{4}\left(A_{x 4}-A_{x p}\right)+C_{5}\left(A_{x 5}-A_{x p}\right)\right)=\left[-\left(\mathrm{C}_{1} \mathrm{~A}_{x 1}+\mathrm{C}_{2} \mathrm{~A}_{x 2}\right.\right. \\
& \left.+\mathrm{C}_{3} \mathrm{~A}_{x 3}+\mathrm{C}_{4} \mathrm{~A}_{x 4}+\mathrm{C}_{5} \mathrm{~A}_{x 5}\right) \\
& \left.+A_{x p}\left(C_{1}+C_{2}+C_{3}+C_{4}+C_{5}\right)\right]
\end{aligned}
$$

By assuming that the current density is constant within the control volume, the $x$ component of the potential in the central node $P$ is given by:

$$
A_{x p}=\frac{1}{C_{p}}\left(\sum_{i=1}^{5} C_{i} A_{x i}-C_{6} \frac{V_{x 2}-V_{x 1}}{d x}+C_{x s}\right)
$$

$C_{p}, C_{1}, C_{2}, C_{3}, C_{4}, C_{5}$ and $C_{6}$, describe geometrical and physical properties associated with the principal control volume and its five neighbourhoods. $V_{x i}$ is the electrical potential. Similarly, we can find both $y$ and $z$ components of the potential in the central node as: 


$$
\begin{aligned}
& A_{y p}=\frac{1}{C_{p}}\left(\sum_{i=1}^{5} C_{i} A_{y i}-C_{6} \frac{V_{y 2}-V_{y 1}}{d y}+C_{y s}\right) \\
& A_{z p}=\frac{1}{C_{p}}\left(\sum_{i=1}^{5} C_{i} A_{z i}-C_{6} \frac{V_{z 2}-V_{z 1}}{d z}+C_{z s}\right)
\end{aligned}
$$

These last three equations can be rewritten in a matrix form as:

$$
\left[\mathbf{M}_{3}\right][\mathbf{A}]=\left[\mathbf{J}_{s}\right]
$$

As in the 2D case, the $3 \mathrm{D}$ FVM method leads to sparse and symmetric matrix $\mathbf{M}_{3}$; this feature is strongly significant regards to computational time and required storage memory especially for the $3 \mathrm{D}$ case. By the way, both $2 \mathrm{D}$ and $3 \mathrm{D}$ computer codes are developed in Matlab. To check the developed computer codes, two TEAM workshop problems and an experimental electromagnetic micro-actuator are analyzed in the next sections.

\section{APPLICATION FOR SOME ELECTROMAGNETIC PROBLEMS} LF

\subsection{TEAM workshop problem No. 30}

The TEAM Workshop problem No.30 deals with a special three phase induction motor, in which the eddy current in the rotor is induced by the time harmonic current in the stator windings, and by the rotation of the rotor [20]. The arrangement of the three phase coils A, B and C is shown in Figure 4. The rotor angular velocity is ranging from 0 to 1200 $\mathrm{rad} / \mathrm{s}$. In this special three phase induction motor, the windings are not embedded in slots and the rotor is made of steel and aluminium with depth of $100 \mathrm{~cm}$. The relative magnetic permeability of the steel is $\mu_{\mathrm{r}}=30$. The motor is supplied with current density $310 \mathrm{~A} / \mathrm{cm}^{2}, 60 \mathrm{~Hz}$.

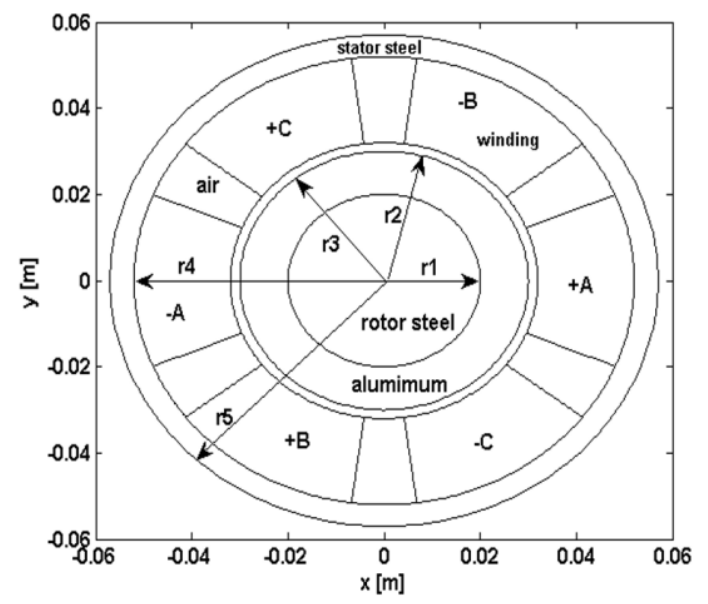

Figure 4. Description of TEAM workshop problem No. 30 $\mathrm{r}_{1}=2, \mathrm{r}_{2}=3, \mathrm{r}_{3}=3.2, \mathrm{r}_{4}=5.2, \mathrm{r}_{5}=5.7, \mathrm{~cm}$

To solve this problem, the developed 2D FVM computer code using triangular mesh is used. Thus, both fundamental quantities of the motor are computed i.e. the electromagnetic torque and the induced voltage in the phase coil $\mathrm{A}$. The electromagnetic torque is calculated by using the following equation:

$$
C_{e m}=\mathbf{r} \times d V \times \mathbf{J}_{\text {indz }} \times \mathbf{B}
$$

Table 1, Figure 5 and Figure 6, illustrate a comparison of the fundamental quantities of the motor, in which we recognize a very small difference between the 2D FVM solution and the analytical solution provided by the TEAM workshop problem. Both results appear to be comparable at all angular velocity of the rotor. Figure 7, Figure 8 and Figure 9 show respectively, the magnetic flux density vectors, the induced current density in the rotor and the electromagnetic force vectors in the rotor at angular velocity of $200 \mathrm{rad} / \mathrm{s}$. As expected, this modelling makes obvious that the finite volume method is a competitive method in the solution of problems related to eddy current and electromagnetic torque computation in the $2 \mathrm{D}$ case.

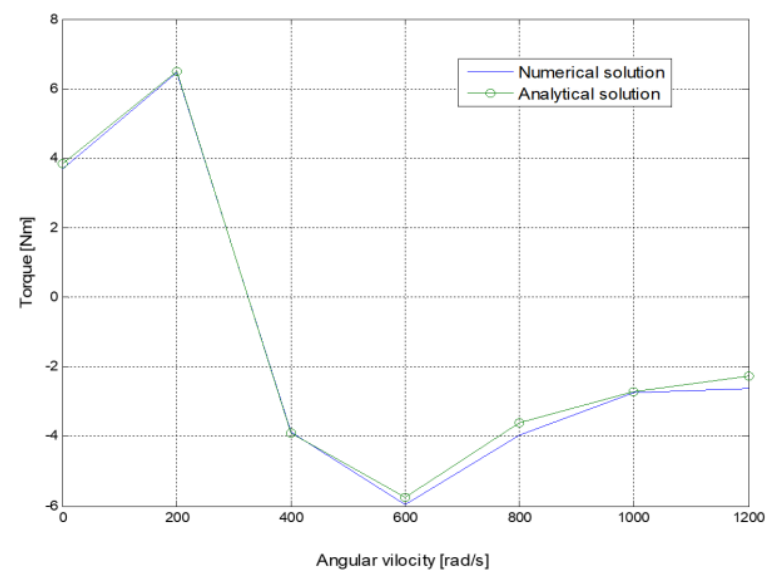

Figure 5. The electromagnetic torque

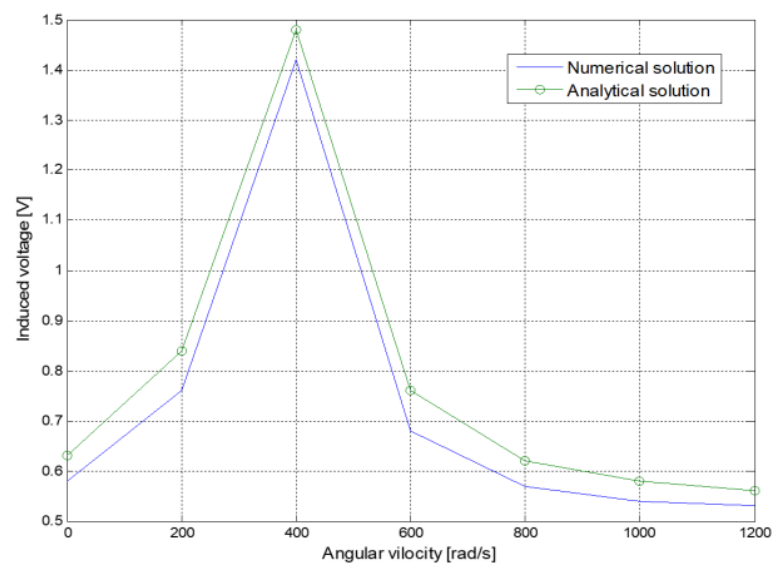

Figure 6. The induced voltage in the phase coil A

\subsection{TEAM workshop problem No. 28}

Figure 10 shows the electromagnetic levitation problem; TEAM workshop problem No. 28 [21]. The problem is made with a cylindrical aluminium plate of electrical conductivity $\sigma=3.4 \mathrm{E} 7 \mathrm{~S}$ and $\mathrm{m}=0.107 \mathrm{~kg}$ placed above two cylindrical coils. The inner coil has 960 turns while the outer coil has 576 turns. Both coils are electrically connected in series, but with inverse sense of winding. By this configuration, the plate is subject to an electromagnetic repulsion force $\mathrm{Fz}$ produced by eddy currents induced in this plate. This electromagnetic force can be expressed as following:

$$
\mathrm{Fz}=\mathbf{J}_{\text {ind }} \times \mathbf{B}
$$


Table 1. Comparison between the FVM solution and the analytical results

\begin{tabular}{ccccc}
\hline & \multicolumn{2}{c}{ Electromagnetic Torque [Nm] } & \multicolumn{2}{c}{ Induced Voltage in The Phase Coil A [V] } \\
Angular Velocity [rad/s] & Analytical Solution & FVM Solution & Analytical Solution & FVM Solution \\
\hline $\mathbf{0}$ & 3.83 & 3.70 & 0.63 & 0.58 \\
$\mathbf{2 0 0}$ & 6.50 & 6.47 & 0.84 & 0.76 \\
$\mathbf{4 0 0}$ & -3.89 & -3.88 & 1.48 & 1.42 \\
$\mathbf{6 0 0}$ & -5.76 & -5.96 & 0.76 & 0.68 \\
$\mathbf{8 0 0}$ & -3.59 & -3.96 & 0.62 & 0.57 \\
$\mathbf{1 0 0 0}$ & -2.70 & -2.73 & 0.58 & 0.54 \\
$\mathbf{1 2 0 0}$ & -2.25 & -2.73 & 0.56 & 0.53 \\
\hline
\end{tabular}

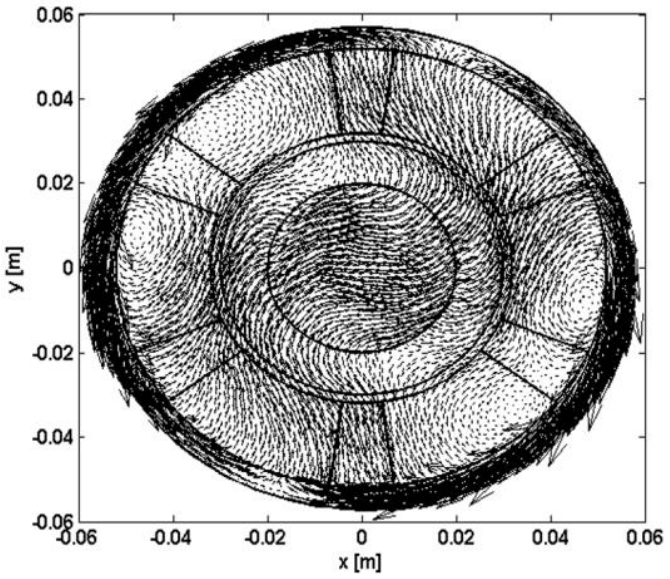

Figure 7. Vectors of the magnetic flux density at 200rad/s

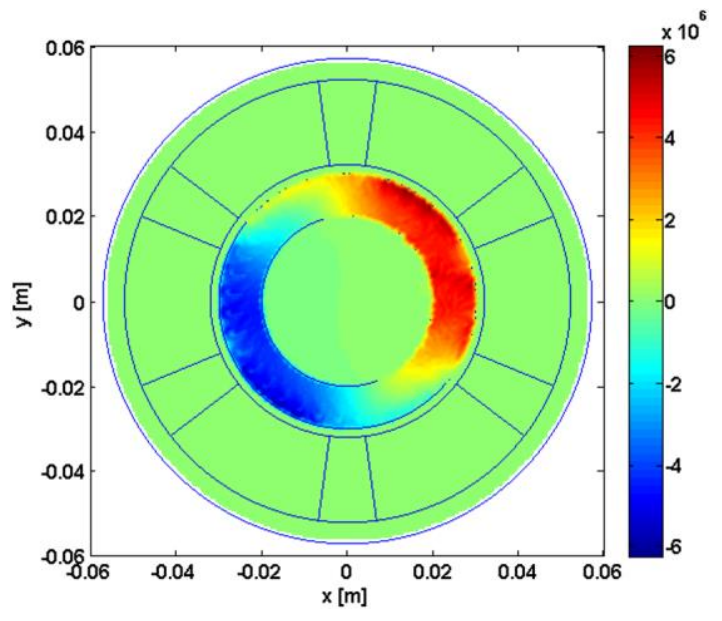

Figure 8. Eddy current density $\left[\mathrm{A} / \mathrm{m}^{2}\right]$ in the rotor at $200 \mathrm{rad} / \mathrm{s}$

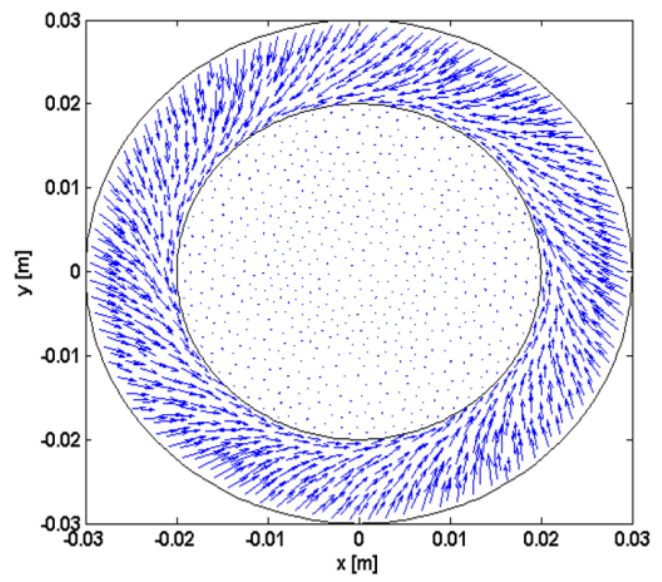

Figure 9. Vectors of the electromagnetic force in the rotor
The aim here is to check the efficiency of the developed 3D FVM computer code associated to the prism mesh, that's why we don't take advantage of the axisymmetrical feature of the problem.

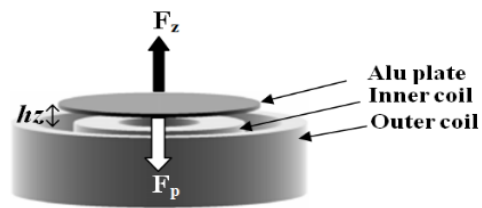

(a)

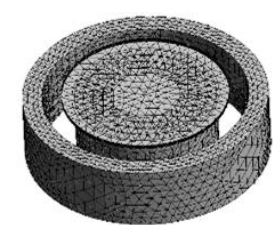

(b)
Figure 10. TEAM workshop problem No. 28 (a) Description, (b) Prism mesh

The solution of TEAM workshop problem No.28 requires the determination of the levitation height $h z$ of the aluminium plate which refers to the distance between the lower edge of the plate and the upper edge of coils. In order to give the equilibrium position of the plate, i.e. equality of both electromagnetic force $\mathrm{Fz}$ and the weight force of the plate $\mathrm{Fp}$, the solution of a correspondent inverse problem is carried out as shown in Figure 11.

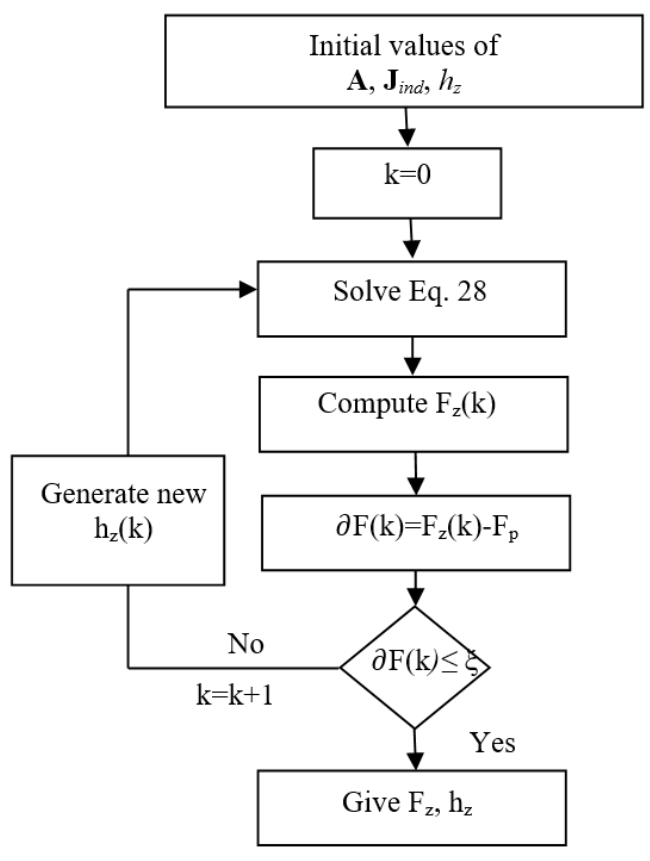

Figure 11. Flowchart of the inverse problem

The algorithm of the inverse problem can be summarized as follows:

Step 0: Give an initial value of the levitation height $h z(\mathrm{k})$, $\mathrm{k}=0$. 
Step 1: By using the direct electromagnetic formula i.e. Eq. 28 , and for a given $h z(\mathrm{k})$ we compute the electromagnetic force $\mathrm{Fz}(\mathrm{k})$ by Eq. 30 .

Step 2: Check the convergence criterion of the inverse problem given by forces difference. If the convergence criterion is satisfied the iteration process is stopped, else the levitation height $h z(\mathrm{k})$ is updated for the next inverse iteration $\mathrm{k}=\mathrm{k}+1$, and return to step (1).

Step 3: The levitation height of the aluminium plate $h z(\mathrm{k})$ and the repulsive force $\mathrm{Fz}(\mathrm{k})$ are given by the last inverse iteration.

Table 2 shows a summary of the results. For each inverse iteration $\mathrm{k}$, both the repulsive electromagnetic force $\mathrm{F} z$ and the levitation height $h z$ are computed. After eight inverse iterations, the difference $\partial \mathrm{F}$ becomes small enough and that is why the iteration process is stopped. As results, the aluminium plate is levitated with $11.84 \mathrm{~mm}$ whereas the experimental value is $11.4 \mathrm{~mm}$ [21].

Table 2. Summary of numerical results obtained by 3D FVM

\begin{tabular}{|c|c|c|}
\hline Inverse iteration $k$ & $\begin{array}{c}\text { Levitation } \\
h z[\mathrm{~mm}]\end{array}$ & $\begin{array}{c}\text { Repulsive force } \\
\text { Fz[N] }\end{array}$ \\
\hline 1 & 15 & 0.59 \\
2 & 7.5 & 2.12 \\
3 & 11.25 & 1.17 \\
4 & 13.13 & 0.84 \\
5 & 12.19 & 0.99 \\
6 & 11.72 & 1.08 \\
7 & 11.95 & 1.03 \\
8 & 11.84 & 1.06 \\
\hline Experimental levitation & 11.4 & \multicolumn{1}{|}{} \\
{$[\mathrm{mm}]$} & \multicolumn{2}{|c}{} \\
\hline
\end{tabular}

As well as in the 2D case, by this we show that the FVM method is a competitive and can be an alternative method in the solution of problems related to eddy current and electromagnetic force computation in the $3 \mathrm{D}$ case.

\subsection{Electromagnetic micro-actuator}

In this section, an experimental electromagnetic microactuator has been analyzed (Figure 12). The micro-actuator is made with two fixed identical coils and two permanent magnets. Each magnet is bonded to a non-magnetic material disc as a magnet support. In addition, the magnets are placed in opposite polarities. For more details on this micro-actuator as dimensions we refer to $[22,23]$.

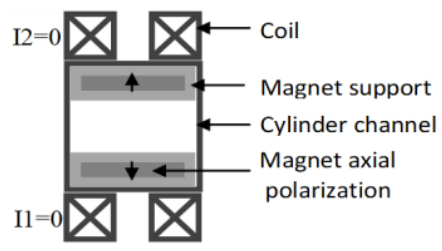

c) Step 3

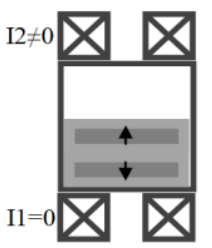

a) Step 1

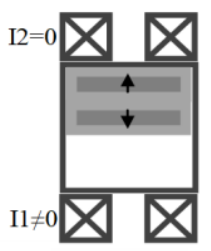

b) Step 2
Figure 12. The actuation steps of the micro-actuator
The actuating mode consists with moving vertically both permanent magnets inside a non-magnetic cylindrical channel. The magnetic force applied to magnets is generated by means the interaction of magnetic fields of both magnets and coils. The actuation is produced with a cyclical movement, where one cycle can be subdivided into three main actuation steps (Figure 12).

Let consider only the actuation of the upper magnet i.e. only the top coil will be supplied. Accordingly, only steps 1 and 3

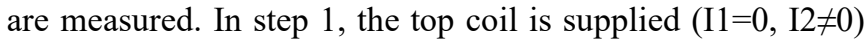
and thus a magnetic repulsive force is applied which drive the upper magnet to reach the lower one. In step 3, both coils are unsupplied $(\mathrm{I} 1=\mathrm{I} 2=0)$ and hence a repulsive force between magnets drive the upper magnet to achieve the upper coil while the lower magnet still fixed to the lower coil.

The aim here is to simulate the actuation mode of the upper magnet by using the FVM method. The magnetic force applied to the upper magnet can be expressed as following:

$$
F_{z}=\int J_{a} \times B_{x}
$$

where, $J a$ is the equivalent fictive current of the upper magnet according to the Ampere's approach:

$$
\mathbf{J}_{a}=\frac{\mathbf{B}_{r} \times \mathbf{n}}{\mu_{0}}
$$

In order to simulate the dynamic characteristic of the upper magnet i.e. magnet displacement versus time, the following mechanical equation is used:

$$
m \cdot \frac{\partial^{2} z}{\partial t^{2}}=F_{z}+m g
$$

where, $m$ and $z$ are respectively the mass and the displacement of the upper magnet, and $g$ is the gravity. Both numerical and experimental characteristics of the actuation are compared in Figure 13. Since frictions are not taken into account in the mechanical equation, the elapsed time in the numerical characteristic is less than in the experimental one with a difference of $4 \mathrm{~ms}$ approximately. However, as in the two previous problems, the FVM method shows once more its effectiveness in the solution of problems related to electrodynamics.

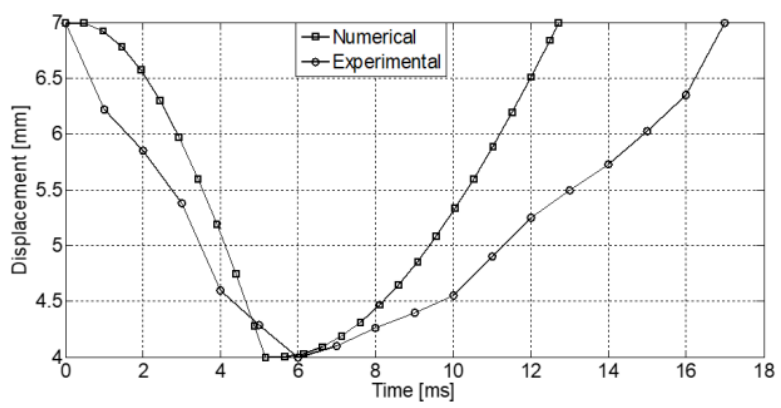

Figure 13. Dynamic characteristic of the upper magnet

\section{CONCLUSION}

This paper presents the FVM as an alternative method for low frequency electromagnetic problems and in particular 
those require eddy current and electromagnetic force or torque computation. Both 2D and 3D computer codes are developed and tested. To examine the efficiency of both programs and showing the competitiveness of the FVM, both TEAM workshop problems No.28 and No.30 and an experimental electromagnetic micro-actuator are analyzed. One can see that numerical results obtained using the FVM are compared to the analytical or experimental results provided by these problems. Furthermore, the 3D FVM associated to the prism mesh can be a very promising for modelling problems without being constrained by their geometries.

\section{REFERENCES}

[1] Balborici, V., Dawson, F.P. (2016). Coupled circuit based representation of piezoelectric structures modeled using the finite volume method. Ultrasonics, 66: 103-110. https://doi.org/10.1016/j.ultras.2015.11.004

[2] Beckstein, P., Galindo, V., Vukčević, V. (2017). Efficient solution of 3D electromagnetic eddy-current problems within the finite volume framework of OpenFOAM. Journal of Computational Physics, 344: 623-646. http://dx.doi.org/10.1016/j.jcp.2017.05.005

[3] Berger, K., Quéval, L., Kameni, A., Alloui, L., Ramdane, B., Trillaud, F., Hell, L.M., Meunier, G., Masson, P., Lévêque, J. (2017). Benchmark on the 3D numerical modeling of a superconducting bulk. Hal.archivesouvertes.fr/hal-01548728v4.

[4] Dong, G., Zou, J., Bayford, R.H., Ma, X., Gao, S., Yan, W., Ge, M. (2005). The comparison between FVM and FEM for EIT forward problem. IEEE Transactions on Magnetics, 41(5): 1468-1471 https//doi.org/10.1109/TMAG.2005.844558

[5] Cheriet, A., Zaoui, A., Feliachi, M., Mimoune, S.M. (2006). Computational performance comparison between FVM and FEM for 3-D magnetostatic problems. NUMELEC'06, 5eme Conférence Européenne sur les Méthodes Numériques en Electromagnétisme, Lille FRANCE, Proc, Art. 47.

[6] Deore, N., Chatterjee, A. (2010). A cell-vertex finite volume time domain method for electromagnetic scattering. Progress in Electromagnetics Research, 12: 115. https//doi.org/10.2528/PIERM10022003

[7] El Ghoul, I., Cheriet, A., Bensaid, S., Lakhdari, A. (2015). FVM model and virtual instrument based system for electromagnetic characterization of steel material. Journal of Applied Engineering Science \& Technology, 2(2): 87-90.

[8] Gong, R., Ruan, J., Chen, J., Quan, Y., Wang, J., Jin, S. (2017). A 3-D coupled magneto-fluid-thermal analysis of a $220 \mathrm{kV}$ three-phase three-limb transformer under DC bias. $\quad$ Energies, $10(4)$ : 422. https://doi.org/10.3390/en10040422

[9] Laguna, A.A., Ozak, N., Lani, A., Deconinck, H., Poedts, S. (2018). Fully-implicit finite volume method for the ideal two-fluid plasma model. Computer Physics Communications, 231: 31-44. http://dx.doi.org/10.1016/j.cpc.2018.05.006

[10] Cioni, J.P., Remaki, M. (1997). Comparaison de deux méthodes de volumes finis en électromagnétisme. Research report No. 3166, INRIA Sophia Antipolis,
France.

[11] Fumeaux, C., Baumann, D., Almpanis, G., Li, E.P., Vahldieck, R. (2008). Finite-volume time-domain method for electromagnetic modelling: Strengths, limitations and challenges. International Journal of Microwave and Optical Technology, 3(3): 318-328.

[12] Davies, J.B., Dean, A.J. (1996). Finite volume with nonuniform mesh for the solution of Maxwell's equations. IEEE Transactions on Magnetics, 32(3): 1417-1420. https//doi.org/10.1109/20.497513

[13] Zou, J., Yuan, J.S., Ma, X.S., Cui, X., Chen, S.M., He, J.L. (2004). Magnetic field analysis of iron-core reactor coils by the finite-volume method. IEEE Transactions on Magnetics, $\quad 40(2)$ : 814-817. https//doi.org/10.1109/TMAG.2004.824773

[14] Cheriet, A., Feliachi, M., Mimoune, S.M. (2007). Nonconforming mesh generation for finite volume method applied to 3-D magnetic field analysis. The European Physical Journal Applied Physics, 37(2): 191195. http://dx.doi.org/10.1051/epjap:2006152

[15] Hamimid, M., Mimoune, S.M., Feliachi, M. (2013). Incorporation of modified dynamic inverse JilesAtherton model in finite volume time domain for nonlinear electromagnetic field computation. Journal of Applied Physics, 113(1): 013915. http://dx.doi.org/10.1063/1.4773535

[16] Cheriet, A., Feliachi, M., Mimoune, S. (2009). 3-D movement simulation technique in FVM method application to eddy current non-destructive testing. COMPEL, 28(1): 77-84. http://dx.doi.org/10.1108/03321640910918887

[17] Li, Y., Torah, R., Beeby, S., Tudor, J. (2012). An allinkjet printed flexible capacitor on a textile using a new poly (4-vinylphenol) dielectric ink for wearable applications. SENSORS, Taipei, pp. 1-4 https://doi.org/10.1155/2012/785271

[18] Mabrouk, A.E.H., Cheriet, A., Feliachi, M., Lakhdari, A.E. (2013). Transient axisymmetric FVM analysis of electodynamic levitation devices. The European Physical Journal Applied Physics, 61(1): 10602. http//doi.org/10.1051/epjap/2013120434

[19] Lepaul, S., Sykulski, J.K., Biddlecombe, C.S., Jay, A.P., Simkin, J. (1999). Coupling of motion and circuits with electromagnetic analysis. IEEE Transactions on Magnetics, 35(3): https//doi.org/10.1109/20.767293

[20] TEAM Benchmark Problems, Problem No.30, Induction Motor Analyses, http://www.compumag.org, accessed on 15 Mar. 2020.

[21] Karl, H., Fetzer, J., Kurz, S., Lehner, G., Rucker, W.M. (1997). Description of TEAM workshop problem 28: An electrodynamic levitation device. Proc. of the TEAM Workshop, Graz, Austria, pp. 48-51.

[22] Amrani, I. Cheriet, A. Bracikowski, N. Feliachi, M., Bekhouche, K. (2017). Design, modeling and fabrication of a novel electromagnetic micro-actuator for a new concept of valveless micropump. JEE, 17(4): 601-608.

[23] Amrani, I., Cheriet, A., Feliachi, M. (2018). Design and experimental investigation of a bi-directional valveless electromagnetic micro-pump. Sensors and Actuators A: Physical, 272 310-317. https://doi.org/10.1016/j.sna.2018.01.065 
MOMENCLATURE

$\mathbf{J}_{\mathrm{s}} \quad$ Source current density

$\mathbf{J}_{\text {ind }} \quad$ Eddy current density

A Magnetic vector potential

B Magnetic flux

$v \quad$ Velocity

$\boldsymbol{C}_{e m} \quad$ Electromagnetic torque

$\mathrm{Fz} \quad$ Electromagnetic force.

$h z \quad$ Levitation height

g Gravity

m Mass

$C_{i} \quad$ Geometrical and physical properties associated with the control volume

\section{Greek symbols}

$\mu \quad$ Magnetic permeability

$\sigma \quad$ Electrical conductivity

$\Omega_{p} \quad$ Principal control volume of node $\mathrm{P}$

$v \quad$ Magnetic reluctivity

\section{Subscripts}

FVM Finite volume method

FEM Finite element method 